\title{
Precompactness of solutions to the Ricci flow in the absence of injectivity radius estimates
}

\author{
DAvid GLickenstein \\ Department of Mathematics, University of California, San Diego \\ 9500 Gilman Drive, La Jolla, CA 92093-0112, USA \\ Email: glicken@math.ucsd.edu
}

\begin{abstract}
Consider a sequence of pointed $n$-dimensional complete Riemannian manifolds $\left\{\left(M_{i}, g_{i}(t), O_{i}\right)\right\}$ such that $t \in[0, T]$ are solutions to the Ricci flow and $g_{i}(t)$ have uniformly bounded curvatures and derivatives of curvatures. Richard Hamilton showed that if the initial injectivity radii are uniformly bounded below then there is a subsequence which converges to an $n$-dimensional solution to the Ricci flow. We prove a generalization of this theorem where the initial metrics may collapse. Without injectivity radius bounds we must allow for convergence in the Gromov-Hausdorff sense to a space which is not a manifold but only a metric space. We then look at the local geometry of the limit to understand how it relates to the Ricci flow.
\end{abstract}

\section{AMS Classification numbers Primary: 53C44}

Secondary: $53 \mathrm{C} 21$

Keywords: Ricci flow, Gromov-Hausdorff convergence

Proposed: Gang Tian

Seconded: John Morgan, Leonid Polterovich
Received: 9 December 2002

Accepted: 10 July 2003 


\section{Introduction}

Consider a solution to the Ricci flow $(\mathcal{M}, g(t))$ defined on a maximal time interval $t \in\left[0, T_{M}\right)$. By Richard Hamilton's work [19] the curvature must go to infinity as $t \rightarrow T_{M}$, but we can look at the limit of dilations of the singularity by taking a sequence of points $x_{i} \in \mathcal{M}$ and times $t_{i} \nearrow T_{M}$ such that

$$
\left|R m\left(x_{i}, t_{i}\right)\right|_{g_{i}\left(t_{i}\right)}=\sup _{x \in \mathcal{M}}\left|R m\left(x, t_{i}\right)\right|_{g_{i}\left(t_{i}\right)} .
$$

We can then consider rescaled solutions to the Ricci flow such as

$$
g_{i}(t)=\left|R m\left(x_{i}, t_{i}\right)\right| g\left(t_{i}+\frac{t}{\left|R m\left(x_{i}, t_{i}\right)\right|}\right)
$$

so that we get a sequence of solutions to the Ricci flow on $\mathcal{M}$ with curvature bounded above and below. We would like to look at the limit of such metrics on $\mathcal{M}$ in order to study what happens as the Ricci flow approaches a singularity. This is referred to as "blowing up about a singularity." Note two things about the blow up:

(1) On a fixed compact time interval the curvatures are bounded.

(2) The diameters may go to infinity.

With these sorts of blow ups in mind Richard Hamilton proved a compactness theorem for solutions to the Ricci flow in [21]. He defines a complete marked solution to the Ricci flow $\left(\mathcal{M}^{n}, g(t), O, \mathcal{F}\right)$ where $\mathcal{M}$ is an $n$-dimensional $C^{\infty}$ manifold, $g(t)$ is a complete Riemannian metric evolving by the Ricci flow on some closed time interval $[0, T], O \in \mathcal{M}$ is a basepoint and $\mathcal{F}$ is a frame at $O$ orthonormal with respect to the metric $g(0)$. The theorem then states that every sequence of complete marked solutions to the Ricci flow $\left\{\left(\mathcal{M}_{i}^{n}, g_{i}(t), O_{i}, \mathcal{F}_{i}\right)\right\}_{i \in \mathbb{N}}$ with uniformly bounded curvature tensors and with injectivity radii of $g_{i}(0)$ at $O_{i}$ uniformly bounded below has a subsequence which converges to a complete marked solution to the Ricci flow $\left(\mathcal{M}_{\infty}^{n}, g_{\infty}(t), O_{\infty}, \mathcal{F}_{\infty}\right)$. Convergence means that for every compact set $K \subset$ $\mathcal{M}_{\infty}$ and for $i$ large enough there are smooth embeddings $\phi_{i}: K \rightarrow \mathcal{M}_{i}$ such that $\phi_{i}^{*}\left(g_{i}\right)$ converges uniformly to $\left.g_{\infty}\right|_{K}$.

We choose the blow up about a singularity so that the curvature bound is immediately satisfied, but the injectivity radius bound is not immediate. For this reason much work has been done to prove that an injectivity radius bound exists for solutions to the Ricci flow. The type I case was studied by Hamilton in [22]. Also the case of type II with bumps of curvature has been studied by Hamilton in [22, Section 23] and Chow-Knopf-Lu in [11]. More recently, 
Perelman [28] proved an injectivity radius estimate for finite time singularities on compact manifolds. In [21, Section 2] Richard Hamilton suggests considering the case where there is no injectivity bounds by looking at the work of Fukaya. This is our main motivation. We also note that Carfora-Marzuoli [3] first used Gromov-Hausdorff convergence as a tool to look at Ricci flow.

Much work has been done studying convergence of Riemannian manifolds, for instance the work by Greene and Wu [17], Peters [30], and Petersen [31] for $C^{k}$ convergence. Hamilton notes, however, that when we are concerned with the Ricci flow much of the hard work is wasted and it is just as easy to work with basic principles. We follow this philosophy. Gromov [18] proved that $n$-dimensional Riemannian manifolds with Ricci curvature bounded below are precompact in the pointed Gromov-Hausdorff topology. Much work has been done to try to understand the collapsing case. Some noteworthy work is that of Cheeger-Gromov [5] and [6] looking at F-structures, Fukaya's fibration theorem for bounded curvature collapse in [13] and [15] generalized to lower curvature bound by Yamaguchi [36], Cheeger-Fukaya-Gromov's development of nilpotent Killing structures in [4], Shioya-Yamaguchi's study of three manifold collapse with lower curvature bound [35], Cheeger-Rong's study of collapse with bounded covering geometry in [8], and work on collapsing with curvature pinched positive by Petrunin-Rong-Tuschmann [32] and Rong [34].

Our work is modelled on Fukaya's study of the local structure of the limit of Riemannian manifolds with a two sided bound on curvature in [14]. These results will be reviewed in Section 2. The following definition is inspired by Fukaya's study of the local structure of limits. Actually, there is a more precise definition that can be given inspired by Peter Petersen's definition of convergence for the noncollapsing case in [31]. In this case we can define a structure on certain metric spaces that are controlled by curvature bounds and how large our submersions are. In this paper we restrict to the simpler case.

Definition 1 A sequence of marked $n$-dimensional Riemannian manifolds $\left\{\left(\mathcal{M}_{i}^{n}, g_{i}, p_{i}\right)\right\}_{i=1}^{\infty}$ locally converges to a pointed metric space $(\mathcal{X}, d, x)$ in the sense of $C^{\infty}$-local submersions at $x$ if there is a Riemannian metric $h$ on an open neighborhood $V \subset \mathbb{R}^{n}$ of 0 , a pseudogroup $\Gamma$ of local isometries of $(V, h)$ such that the quotient is well defined, an open set $U \subset \mathcal{X}$, and maps $\phi_{i}:(V, 0) \rightarrow\left(\mathcal{M}_{i}, p_{i}\right)$ such that

(1) $\left\{\left(\mathcal{M}_{i}, d_{g_{i}}, p_{i}\right)\right\}_{i=1}^{\infty}$ converges to $(\mathcal{X}, d, x)$ in the pointed Gromov-Hausdorff topology,

(2) the identity component of $\Gamma$ is a Lie group germ, 
(3) $\left(V / \Gamma, \bar{d}_{h}\right)$ is isometric to $(U, d)$,

(4) $\left(\phi_{i}\right)_{*}$ is nonsingular on $V$ for all $i \in \mathbb{N}$, and

(5) $h$ is the $C^{\infty}$ limit of $\phi_{i}^{*} g_{i}$ (uniform convergence on compact sets together with all derivatives)

where $\bar{d}_{h}$ is the induced distance in the quotient.

We use the term convergence in $C^{\infty}$-local submersions since there is actually a local submersion (actually a local covering) structure on manifolds with bounded curvature defined by the exponential map. The convergence is really of these structures. A local isometry $f$ of $V$ is a map from $V$ to possibly a larger space which is a Riemannian isometry, i.e. $f^{*} h=h$, usually for us it will be an injective map $B(p, r / 2) \rightarrow B(p, r)$ for some $p \in V$ and some $r>0$. The global definition is:

Definition 2 A sequence of marked $n$-dimensional Riemannian manifolds $\left\{\left(\mathcal{M}_{i}^{n}, g_{i}, p_{i}\right)\right\}_{i=1}^{\infty}$ converges to a pointed metric space $(\mathcal{X}, d, x)$ in the sense of $C^{\infty}$-local submersions if for every $y \in \mathcal{X}$ there exist $q_{i} \in \mathcal{M}_{i}$ such that $\left\{\left(\mathcal{M}_{i}, g_{i}, q_{i}\right)\right\}_{i=1}^{\infty}$ locally converges to $(\mathcal{X}, d, y)$ in the sense of $C^{\infty}$-local submersions at $y$.

For the explanation of notation and definition of some of the terms, we refer the reader to Section 2. We are now ready to state our main theorem. We refer to the metric $h$ on the ball $V$ corresponding to the point $y \in \mathcal{X}$ as $h_{y}$.

Theorem 3 (Main Theorem) Let $C_{k}>0$ be constants for $k \in \mathbb{N}$ and $\left\{\left(\mathcal{M}_{i}^{n}\right.\right.$, $\left.\left.g_{i}(t), p_{i}\right)\right\}_{i=1}^{\infty}$, where $t \in[0, T]$, be a sequence of marked solutions to the Ricci flow on complete manifolds such that

$$
\left|R m\left(g_{i}(t)\right)\right|_{g_{i}(t)} \leq 1
$$

and

$$
\left|D_{g_{i}(t)}^{k} R m\left(g_{i}(t)\right)\right|_{g_{i}(t)} \leq C_{k}
$$

for all $i, k \in \mathbb{N}$ and $t \in[0, T]$.

Then there is a subsequence $\left\{\left(\mathcal{M}_{i_{k}}, g_{i_{k}}(t), p_{i_{k}}\right)\right\}_{k=1}^{\infty}$ and a one parameter family of complete pointed metric spaces $(\mathcal{X}, d(t), x)$ such that for each $t \in[0, T]$, $\left(\mathcal{M}_{i_{k}}, d_{g_{i_{k}}(t)}, p_{i_{k}}\right)$ converges to $\left(\mathcal{X}_{\infty}, d_{\infty}(t), x_{\infty}\right)$ in the sense of $C^{\infty}$-local submersions and the metrics $h_{y}(t)$ are solutions to the Ricci flow equation. 
Peng Lu [27] has generalized Hamilton's compactness theorem in another direction. He extended it to convergence of sequences of orbifold solutions to the Ricci flow and showed that under similar conditions to Hamilton there is a subsequence which converges to a orbifold solution to the Ricci flow. That theorem uses an analogue of the injectivity radius bound. Although not treated here, this work should be able to be extended to a larger class of sequences such as orbifolds or potentially more general structures.

The primary advantage of the main theorem over other compactness theorems is that it requires no control on the injectivity radius at all. Hence if we consider blow ups about singularities as done in [22], we do not have to worry about injectivity radius bounds, but may form singularity models which collapse as well as ones which do not collapse. In many cases it is believed that sequences arising from singularity blowups will not collapse. Recent work of Perelman [28] shows that sequences arising from blow ups of finite time singularities on closed manifolds cannot collapse. A study of collapsing singularity models may be useful in proving other types of singularities, for instance Type IIb singularities, cannot collapse. In [10] Chow, Lu, and the author use the main theorem to classify singularity models of three-dimensional manifolds arising from sequences with almost nonnegative sectional curvature and diameters tending to infinity. In addition, virtual limits are constructed to gain geometric insight on the limits of sequences which collapse to non-manifold or low dimensional limits. We hope to be able to use this theorem to better understand the relationship between injectivity radius and the Ricci flow for infinite time singularities.

We hope to be able to use the compactness theorem to understand long time behavior of the Ricci flow analytically. Many nonsingular solutions collapse. Isenberg-Jackson [24] showed that some homogeneous metrics collapse. Carafora-Isenberg-Jackson [2], Hamilton-Isenberg [23], Knopf [25], and KnopfMcLeod [26] demonstrated families of metrics which quasiconverge to homogeneous solutions, which have the same asymptotic behavior and hence collapse.

Perelman's work ([28] and [29]) indicates a decomposition of three-manifolds into "thick" and "thin" parts. The thin parts are collapsing and can be understood topologically as a graph manifold. We hope to be able to use a compactness theorem to understand the geometry of these parts. A greater understanding of the manifolds or parts of the manifolds may allow for the definition of a weak solution of the Ricci flow, defined in terms of local submersions as in the compactness theorem. This could be extremely useful in the study of manifolds of any dimension. 
Acknowledgements The author would like to thank his advisor Ben Chow for suggesting this problem and for all of his help and support. The author would also like to thank Nolan Wallach for all of his help.

The author was partially supported by NSF grant DMS-0203926.

\section{Notation and Background}

For future use we would like to establish the following notation. A pointed map written as $\phi:(X, x) \rightarrow(Y, y)$ indicates that $\phi(x)=y$. If $\left(X, g_{X}\right)$ and $\left(Y, g_{Y}\right)$ are Riemannian manifolds, then $\phi$ is a local isometry if $\phi^{*} g_{Y}=g_{X}$. For any Riemannian manifold $(M, g)$ we use $d_{g}$ to denote the distance function on $M$ determined by $g$ by integration along paths that makes $\left(M, d_{g}\right)$ into a metric space. To avoid confusion, the term "distance function" shall be used to refer to the metric for the metric space structure, and the term "metric" shall be reserved for Riemannian metrics. We also introduce the following notation for balls in $(X, d)$

$$
B_{d}(p, r) \doteqdot\{q \in X: d(p, q)<r\} .
$$

When it is clear which is the correct distance function, we may use $B_{X}(p, r)$ instead. Given $Y \subset X$ we can define the ball around $Y$ as

$$
B_{d}(Y, r) \doteqdot\{q \in X: \exists y \in Y \text { such that } d(y, q)<r\} \text {. }
$$

When no distance is specified, for instance $B(0, r)$, it is assumed that we mean the Euclidean ball in $\mathbb{R}^{n}$. We also shall sometimes omit 0 in the Euclidean ball and use $B(r) \doteqdot B(0, r)$.

For the following we refer the reader to the treatment of local groups in [33]. We prefer the term pseudogroup since the term local group usually refers to equivalence classes of arbitrarily small neighborhoods of the identity. We shall actually need some of the components of our pseudogroup, so group germs are not sufficient.

Definition 4 A topological space $G$ is a pseudogroup if for certain pairs of elements $a, b \in G$ there is a product $a b \in G$ such that

(1) If $a b,(a b) c, a(b c)$ are all defined, then $(a b) c=a(b c)$

(2) If $a b$ is defined then for every neighborhood $W$ of $a b$ there exist neighborhoods $U$ of $a$ and $V$ of $b$ such that $x y$ is defined for all $x \in U$ and $y \in V$ and $x y \in W$. 
(3) There is an identity element $e$ such that if $a \in G$ then $a e$ is defined and $a e=a$.

(4) If for a pair of elements $a, b \in G, a b$ is defined and $a b=e$, then $a$ is a left inverse for $b$ and we say $a=b^{-1}$. If $b$ has a left inverse, then for every neighborhood $U$ of $b^{-1}$ there exists a neighborhood $V$ of $b$ such that every element $y \in V$ possesses a left inverse $y^{-1} \in U$.

It is easy to show that if a pseudogroup $G$ acts as $B(p, r / 2) \rightarrow B(p, r)$ as local isometries such that the relation $x \sim \gamma x$ for all $\gamma \in G$ is an equivalence relation for $x \in B(p, r / 4)$ then the quotient $B(p, r / 4) / G$ is well-defined. When a pseudogroup acts in this way, we can define the push forward of the distance function, denoted $\bar{d}$, which is a distance function on the quotient. It is defined as

$$
\bar{d}([x],[y])=\inf \left\{d\left(\gamma x, \gamma^{\prime} y\right): \gamma, \gamma^{\prime} \in G\right\}
$$

where $[x]$ is the equivalence class of $x$.

We can also define Lie group germ as a special case of a pseudogroup.

Definition 5 A pseudogroup $G$ is a Lie group germ if a neighborhood of the identity of $G$ can be given a differentiable structure such that group multiplication and inversion are differentiable maps when defined.

Essentially this definition says that the pseudogroup $G$ is a subspace of a Lie group which may not be a subgroup, i.e. closed under group operations.

Recall the definition of pointed Gromov-Hausdorff distance. Our definition is the one used in [14]. We first need the notion of an $\epsilon$-pointed GromovHausdorff approximation. Note that since a major application is the blow up of a singularity and in that case the diameters may go to infinity, we shall always work in the pointed category.

Definition 6 Let $\left(X, d_{X}\right)$ and $\left(Y, d_{Y}\right)$ be metric spaces with $x_{0} \in X$ and $y_{0} \in Y$. A map $f:\left(X, x_{0}\right) \rightarrow\left(Y, y_{0}\right)$ is an $\varepsilon$-pointed Gromov-Hausdorff approximation if it satisfies the following:

- $B_{Y}\left(y_{0}, 1 / \varepsilon-\varepsilon\right) \subseteq B_{Y}\left(f\left[B_{X}\left(x_{0}, 1 / \varepsilon\right)\right], \varepsilon\right)$

- For all $x, x^{\prime} \in B_{X}\left(x_{0}, 1 / \varepsilon\right)$ we have

$$
\left|d_{X}\left(x, x^{\prime}\right)-d_{Y}\left(f(x), f\left(x^{\prime}\right)\right)\right|<\epsilon
$$


Note that $f$ is not required to be continuous. The first condition essentially says $f$ maps $X$ to almost all of $Y$, in the pointed sense, and the second condition says that $f$ is approximately an isometry. Let $\vec{X}$ and $\vec{Y}$ respectively denote the pointed metric spaces $\left(X, d_{X}, x_{0}\right)$ and $\left(Y, d_{Y}, y_{0}\right)$. We can now define the pointed Gromov-Hausdorff distance $d_{G H}$ between $\vec{X}$ and $\vec{Y}$ as:

$$
d_{G H}(\vec{X}, \vec{Y}) \doteqdot \inf \left\{\epsilon \mid \begin{array}{c}
\exists \epsilon \text {-pointed Gromov-Hausdorff } \\
\text { approximations } f:\left(X, x_{0}\right) \rightarrow\left(Y, y_{0}\right) \\
\text { and } g:\left(Y, y_{0}\right) \rightarrow\left(X, x_{0}\right)
\end{array}\right\} .
$$

We shall sometimes omit the dependence on the distance function if it is clear what we mean. Gromov-Hausdorff distance is especially interesting since it can compare manifolds with different topologies.

Note that it is not a distance in the usual sense, since the triangle inequality does not hold exactly (see Proposition 7 ). We have the following useful propositions most of whose proofs we omit here. For further details see [16]. The first is an approximate triangle inequality, which allows us to treat $d_{G H}$ essentially as a distance.

Proposition 7 Let $\overrightarrow{\mathcal{X}}_{i} \doteqdot\left(\mathcal{X}_{i}, d_{i}, O_{i}\right)$ be pointed metric spaces for $i=1,2,3$. If $d_{G H}\left[\overrightarrow{\mathcal{X}}_{1}, \overrightarrow{\mathcal{X}}_{2}\right] \leq 1 / 2$ and $d_{G H}\left[\overrightarrow{\mathcal{X}}_{2}, \overrightarrow{\mathcal{X}}_{3}\right] \leq 1 / 2$ then

$$
d_{G H}\left[\overrightarrow{\mathcal{X}}_{1}, \overrightarrow{\mathcal{X}}_{3}\right] \leq 2\left(d_{G H}\left[\overrightarrow{\mathcal{X}}_{1}, \overrightarrow{\mathcal{X}}_{2}\right], d_{G H}\left[\overrightarrow{\mathcal{X}}_{2}, \overrightarrow{\mathcal{X}}_{3}\right]\right) \text {. }
$$

The following is an associativity of convergence.

Proposition 8 Let $\overrightarrow{\mathcal{X}}_{i} \doteqdot\left(\mathcal{X}_{i}, d_{i}, O_{i}\right)$ and $\overrightarrow{\mathcal{X}}_{i}^{\prime} \doteqdot\left(\mathcal{X}_{i}^{\prime}, d_{i}^{\prime}, O_{i}^{\prime}\right)$ be pointed metric spaces for $i \in \mathbb{N} \cup\{\infty\}$. If $d_{G H}\left[\overrightarrow{\mathcal{X}}_{i}, \overrightarrow{\mathcal{X}}_{i}^{\prime}\right] \leq \varepsilon$ for each $i \in \mathbb{N}$ and if $\overrightarrow{\mathcal{X}}_{i}$ converges to $\overrightarrow{\mathcal{X}}_{\infty} \doteqdot\left(\mathcal{X}_{\infty}, d_{\infty}, O_{\infty}\right)$ and $\overrightarrow{\mathcal{X}}_{i}^{\prime}$ converges to $\overrightarrow{\mathcal{X}}_{\infty}^{\prime} \doteqdot\left(\mathcal{X}_{\infty}^{\prime}, d_{\infty}^{\prime}, O_{\infty}^{\prime}\right)$ in GH then $d_{G H}\left[\overrightarrow{\mathcal{X}}_{\infty}, \overrightarrow{\mathcal{X}}_{\infty}^{\prime}\right] \leq 4 \varepsilon$

The following is an interaction between Gromov-Hausdorff convergence and Lipschitz convergence.

Proposition 9 Let $\left(\mathcal{X}_{i}, d_{i}, x_{i}\right)$ and $\left(\mathcal{X}_{i}^{\prime}, d_{i}^{\prime}, x_{i}^{\prime}\right)$ be pointed complete length spaces (for details see [1]) for $i \in \mathbb{N} \cup\{\infty\}$. If for each $i \in \mathbb{N}$ there is a homeomorphism $\mathcal{X}_{i} \rightarrow \mathcal{X}_{i}^{\prime}$ taking $x_{i}$ to $x_{i}^{\prime}$ and Lipschitz with Lipschitz constant $c<\infty$ (independent of $i$ ) and if $\left(\mathcal{X}_{i}, d_{i}, x_{i}\right)$ converges to $\left(\mathcal{X}_{\infty}, d_{\infty}, x_{\infty}\right)$ and $\left(\mathcal{X}_{i}^{\prime}, d_{i}^{\prime}, x_{i}^{\prime}\right)$ converges to $\left(\mathcal{X}_{\infty}^{\prime}, d_{\infty}^{\prime}, x_{\infty}^{\prime}\right)$ in the pointed Gromov-Hausdorff topology then there is a Lipschitz homeomorphism $\left(\mathcal{X}_{\infty}, x_{\infty}\right) \rightarrow\left(\mathcal{X}_{\infty}^{\prime}, x_{\infty}^{\prime}\right)$ with Lipschitz constant $c$. 
The proof of Proposition 9 relies heavily on the following fact.

Proposition 10 If $\mathcal{X}$ is a length space, then $\mathcal{X}$ is isometric to the direct limit

$$
\underset{\lim }{\longrightarrow} B_{\mathcal{X}}(p, 1 / i)
$$

where the structure is just the obvious inclusions.

As a corollary we get the following important property of length spaces.

Proposition 11 If $\left(\mathcal{X}_{i}, d_{i}, x_{i}\right)$ are length spaces converging to $\left(\mathcal{X}_{\infty}, d_{\infty}, x_{\infty}\right)$ in the pointed Gromov-Hausdorff topology, then for every $\rho>0, B_{d_{i}}\left(x_{i}, \rho\right)$ converge to $B_{d_{\infty}}\left(x_{\infty}, \rho\right)$ in the pointed Gromov-Hausdorff topology.

The following relates equivalence of Riemannian metrics with Gromov-Hausdorff distance.

Proposition 12 Let $g$ and $h$ be Riemannian metrics on a smooth manifold $\mathcal{M}$ with $O \in \mathcal{M}$. Suppose there exist $\delta>0$ such that

$$
(1+\delta)^{-1} g \leq h \leq(1+\delta) g,
$$

then

$$
d_{G H}\left[\left(\mathcal{M}, d_{g}, O\right),\left(\mathcal{M}, d_{h}, O\right)\right]<2 \delta^{1 / 4}(1+\delta)^{1 / 2} .
$$

Finally the following indicates how we can change basepoints.

Proposition 13 If $\left(\mathcal{X}_{i}, d_{i}, x_{i}\right)$ converges to $\left(\mathcal{X}_{\infty}, d_{\infty}, x_{\infty}\right)$ in the pointed Gromov-Hausdorff distance, then for every $y_{\infty} \in \mathcal{X}_{\infty}$ there exist $y_{i} \in \mathcal{X}_{i}$ such that $\left(\mathcal{X}_{i}, d_{i}, y_{i}\right)$ converges to $\left(\mathcal{X}_{\infty}, d_{\infty}, y_{\infty}\right)$ in the pointed Gromov-Hausdorff distance.

Gromov proved the following precompactness result about Riemannian manifolds with the Gromov-Hausdorff topology.

Theorem 14 [18, Theorem 5.3] The set of $n$-dimensional pointed Riemannian manifolds with Ricci curvature uniformly bounded below is precompact with respect to the pointed Gromov-Hausdorff topology.

This says that every sequence has a subsequence which converges to a complete metric space. The space is actually a length space with curvature bounded below in the sense of Alexandrov (see [1]).

In [14] Fukaya made the definition of a smooth element of the closure of the set of Riemannian manifolds, but since smooth has a different meaning in our case, we instead use the term nice. His definition is the following. 
Definition 15 A pointed metric space $(X, p)$ is called nice if there exists

- a neighborhood $U$ of $p$ in $X$

- a compact Lie group $G_{p}$, whose identity component is isomorphic to a torus

- a faithful representation of $G_{p}$ into $O(m)$

such that $U$ is homeomorphic to $V / G_{p}$ for some neighborhood $V$ of 0 in $\mathbb{R}^{m}$ (note that this is a linear action of a subgroup of the orthogonal group on $\left.\mathbb{R}^{m}\right)$ and furthermore there is a $G_{p}$-invariant Riemannian metric $g$ such that $\left(V / G_{p}, \overline{0}\right)$ with distance function $\bar{d}_{g}$ is isometric (as pointed metric spaces) to $(U, p)$. (Here we used $\overline{0}$ to denote the equivalence class of 0 and $\bar{d}_{g}$ to denote the induced metric on the quotient space.)

We note that $m$ may not equal $n$. In Theorem 0.5 Fukaya proved that nice metric spaces are dense in the closure of $n$-dimensional Riemannian manifolds with uniformly bounded curvature. Upon close inspection of the proof, however, he proves the following theorem, not explicitly stated, which is exactly the unparameterized version of our local theory.

Theorem 16 If $\left\{\mathcal{M}_{i}, g_{i}, O_{i}\right\}_{i \in \mathbb{N}}$ is a sequence of $n$-dimensional pointed Riemannian manifolds such that

$$
\left|R m\left(g_{i}\right)\right|_{g_{i}} \leq 1
$$

and there exist $C_{k}>0$ such that

$$
\left|D_{i}^{k} R m\left(g_{i}\right)\right|_{g_{i}} \leq C_{k}
$$

then there exists a subsequence which converges to a metric space $\left(\mathcal{X}, d, x_{0}\right)$ such that there exists a Riemannian metric $h$ on $B(0,1) \subset \mathbb{R}^{n}$, a pseudogroup $G_{\infty}$ of local isometries acting as $B(0,1 / 2) \rightarrow B(0,1)$ such that $\left(B_{\mathcal{X}}\left(x_{0}, 1 / 4\right), d\right)$ is isometric to $\left(B(0,1 / 4) / G_{\infty}, \bar{d}_{h}\right)$.

Furthermore there is some characterization of $G_{\infty}$; it is a local Lie group with nilpotent Lie algebra and $G_{x_{0}}$ as in the definition of "nice" is the stabilizer of 0 , i.e. the subgroup (not only subpseudogroup) $\left\{\gamma \in G_{\infty}: \gamma(0)=0\right\}$.

We also need the following estimates of Hamilton.

Lemma 17 [21, Lemma 2.4] Let $M$ be a Riemannian manifold with metric $G, K$ a compact subset of $M$, and $G_{k}$ a collection of solutions to the Ricci flow 
defined on neighborhoods of $K \times[\beta, \psi]$ with the time interval $[\beta, \psi]$ containing $t=0$. Let $D$ denote covariant derivative with respect to $G$ and $\mid$ the length of a tensor with respect to $G$, while $D_{k}$ and ||$_{k}$ are the same for $G_{k}$. Suppose that

(1) the metrics $G_{k}$ are all uniformly equivalent to $G$ at $t=0$ on $K$, so that

$$
c G(V, V) \leq G_{k}(V, V) \leq C G(V, V)
$$

for some constants $c>0$ and $C<\infty$ independent of $k$; and

(2) the covariant derivatives of the metrics $G_{k}$ with respect to the metric $G$ are all uniformly bounded at $t=0$ on $K$, so that

$$
\left|D^{p} G_{k}\right| \leq C_{p}
$$

for some constants $C_{p}<\infty$ independent of $k$ for all $p \geq 1$; and

(3) the covariant derivatives of the curvature tensors $R m_{k}$ of the metrics $G_{k}$ are uniformly bounded with respect to the $G_{k}$ on $K \times[\beta, \psi]$, so that

$$
\left|D_{k}^{p} R m_{k}\right|_{k} \leq C_{p}^{\prime}
$$

for some constants $C_{p}^{\prime}$ independent of $k$ for all $p \geq 0$.

Then the metrics $G_{k}$ are uniformly bounded with respect to $G$ on $K \times[\beta, \psi]$, so that

$$
\tilde{c} G(V, V) \leq G_{k}(V, V) \leq \widetilde{C} G(V, V)
$$

for some constant $\tilde{c}$ and $\widetilde{C}$ independent of $k$, and the covariant derivatives of the metrics $G_{k}$ with respect to the metric $G$ are uniformly bounded on $K \times[\beta, \psi]$, so that

$$
\left|D^{p} G_{k}\right| \leq \widetilde{C}_{p}
$$

for some constants $\widetilde{C}_{p}$ independent of $k$ with $\tilde{c}, \widetilde{C}$ and $\widetilde{C}_{p}$ depending only on $c, C, C_{p}$ and $C_{p}^{\prime}$ and the dimension.

\section{Ricci flow estimates}

In this section we prove some estimates for ball sizes under evolution by the Ricci flow. These are improvements of Hamilton's lemma given above as Lemma 17. The ideas are wholly within Hamilton's work, but we reproduce them here. Assume

$$
\left|R m\left(g_{i}(t)\right)\right|_{g_{i}(t)} \leq C_{0}
$$


and let $R c_{i}(t) \doteqdot R c\left(g_{i}(t)\right)$. Let $V \in T_{p} \mathcal{M}$. We estimate $g_{i}\left(t_{0}\right)-g_{i}(t)$ :

$$
\begin{aligned}
{\left[g_{i}(t)-g_{i}\left(t_{0}\right)\right][V, V] } & =\int_{t_{0}}^{t} \frac{\partial}{\partial s} g_{i}(s)[V, V] d s \\
& =\int_{t_{0}}^{t}-2 R c_{i}(s)[V, V] d s .
\end{aligned}
$$

and

$$
-\left|R c_{i}(t)\right| g_{i}(t) \leq R c_{i}(t) \leq\left|R c_{i}(t)\right| g_{i}(t)
$$

And by our bounds on curvature,

$$
\left|R c_{i}(t)[V, V]\right| \leq C_{0} g_{i}(t)[V, V] .
$$

Then we have

$$
\left|\frac{\partial}{\partial t} \log \left(g_{i}(t)[V, V]\right)\right|=2 \frac{\left|R c_{i}(t)[V, V]\right|}{g_{i}(t)[V, V]} \leq 2 C_{0}
$$

so we can estimate

$$
\begin{aligned}
\left|\log \left(g_{i}(t)[V, V]\right)-\log \left(g_{i}\left(t_{0}\right)[V, V]\right)\right| & =\left|\int_{t_{0}}^{t} \frac{\partial}{\partial t} \log \left(g_{i}(s)[V, V]\right) d s\right| \\
& \leq \int_{t_{0}}^{t}\left|\frac{\partial}{\partial t} \log \left(g_{i}(s)[V, V]\right)\right| d s \\
& \leq 2 C_{0}\left(t-t_{0}\right) .
\end{aligned}
$$

We conclude that

$$
-2 C_{0}\left(t-t_{0}\right) \leq \log \left(g_{i}(t)[V, V]\right)-\log \left(g_{i}\left(t_{0}\right)[V, V]\right) \leq 2 C_{0}\left(t-t_{0}\right)
$$

which we can rewrite as

$$
e^{-2 C_{0}\left(t-t_{0}\right)} \leq \frac{g_{i}(t)[V, V]}{g_{i}\left(t_{0}\right)[V, V]} \leq e^{2 C_{0}\left(t-t_{0}\right)} .
$$

Since $e^{x}=1+\delta(x)$ where $\delta(x) \rightarrow 0$ as $x \rightarrow 0$, we also have

$$
\left|g_{i}(t)[V, V]-g_{i}\left(t_{0}\right)[V, V]\right| \leq \delta\left(t-t_{0}\right)
$$

We can use this to estimate distances as well by integrating over paths and get the following lemma.

Lemma 18 Suppose $(\mathcal{M}, g(t))$ are solutions to the Ricci flow for $t \in[0, T)$ such that the sectional curvatures are uniformly bounded by $C_{0}$. Then we have the following:

Geometry $8 \mathcal{T}$ Topology, Volume 7 (2003) 
(1) For all vectors $V$ and $t, t_{0} \in[\alpha, \omega)$

$$
e^{-2 C_{0}\left|t-t_{0}\right|} \leq \frac{g(t)[V, V]}{g\left(t_{0}\right)[V, V]} \leq e^{2 C_{0}\left|t-t_{0}\right|}
$$

(2) For all $\delta>0$ there exists an $\eta>0$ such that if $\left|t-t_{0}\right|<\eta$ then

$$
-\delta g\left(t_{0}\right) \leq g(t)-g\left(t_{0}\right) \leq \delta g\left(t_{0}\right) .
$$

(3) For all $\delta>0$ there exists an $\eta>0$ such that if $\left|t-t_{0}\right|<\eta$ then

$$
\left|d_{g(t)}\left(q, q^{\prime}\right)-d_{g\left(t_{0}\right)}\left(q, q^{\prime}\right)\right| \leq \delta^{1 / 2} d_{g\left(t_{0}\right)}\left(q, q^{\prime}\right)
$$

for all $q, q^{\prime} \in \mathcal{M}$.

Proof Use the above arguments and let $\delta=\exp \left(2 C_{0}\left|t-t_{0}\right|\right)-1$ and $\eta=$ $\log (\delta+1) /\left(2 C_{0}\right)$.

As a corollary we can compute how the size of balls change with the Ricci flow.

Proposition 19 If $g(t)$ is a solution to the Ricci flow such that the curvature is bounded by the constant 1 then for all $\rho>0$

$$
\begin{aligned}
& B_{g(t)}(0, r(t) \rho) \subset B_{g(0)}(0, \rho) \\
& B_{g(0)}(0, r(t) \rho) \subset B_{g(t)}(0, \rho)
\end{aligned}
$$

where

$$
r(t)=\frac{1}{1+\left(e^{2 t}-1\right)^{1 / 2}}
$$

Proof Our estimates in Lemma 18 show

$$
\left|d_{g(0)}\left(q, q^{\prime}\right)-d_{g(t)}\left(q, q^{\prime}\right)\right| \leq \delta(t)^{1 / 2} d_{g(t)}\left(q, q^{\prime}\right)
$$

where $\delta(t)=\left(e^{2 t}-1\right)$, so

$$
\begin{aligned}
d_{g(0)}\left(p_{i}, q\right) & \leq\left|d_{g(0)}\left(p_{i}, q\right)-d_{g(t)}\left(p_{i}, q\right)\right|+d_{g(t)}\left(p_{i}, q\right) \\
& \leq\left(1+\delta(t)^{1 / 2}\right) d_{g(t)}\left(p_{i}, q\right)
\end{aligned}
$$

so $B_{g(t)}\left(0,\left(1+\delta(t)^{1 / 2}\right)^{-1} \rho\right) \subset B_{g(0)}(0, \rho)$. Recall that we define

$$
r(t) \doteqdot \frac{1}{1+\left(e^{2 t}-1\right)^{1 / 2}},
$$

Geometry ${ }^{6}$ Topology, Volume 7 (2003) 
so this says $B_{g(t)}(0, r(t) \rho) \subset B_{g(0)}(0, \rho)$. Conversely, we have

$$
\begin{aligned}
d_{g(t)}\left(p_{i}, q\right) & \leq\left|d_{g(t)}\left(p_{i}, q\right)-d_{g(0)}\left(p_{i}, q\right)\right|+d_{g(0)}\left(p_{i}, q\right) \\
& \leq\left(1+\delta(t)^{1 / 2}\right) d_{g(0)}\left(p_{i}, q\right)
\end{aligned}
$$

so $B_{g(0)}(0, r(t) \rho) \subset B_{g(t)}(0, \rho)$.

\section{Convergence of metric spaces}

In this section we prove that the metric spaces converge. We will prove a slightly more general theorem, since only the continuity in the $t$ variable of solutions of the Ricci flow is used.

Theorem 20 Let $\left\{\left(\mathcal{M}_{i}, g_{i}(t), p_{i}\right)\right\}_{i=1}^{\infty}$, where $t \in[0, T]$, be a sequence of pointed Riemannian manifolds of dimension $n$ which is continuous in the $t$ variable in the following way: for each $\delta>0$ there exists $\eta>0$ such that if $t_{0}, t_{1} \in[0, T]$ satisfies $\left|t_{0}-t_{1}\right|<\eta$ then

$$
(1+\delta)^{-1} g_{i}\left(t_{0}\right) \leq g_{i}\left(t_{1}\right) \leq(1+\delta) g_{i}\left(t_{0}\right)
$$

for all $i>0$, and such that

$$
R c\left(g_{i}(t)\right) \geq c g_{i}(t)
$$

where $c$ does not depend on $t$ or $i$.

Then there is a subsequence $\left\{\left(\mathcal{M}_{i_{k}}, g_{i_{k}}(t), p_{i_{k}}\right)\right\}_{k=1}^{\infty}$ and a 1-parameter family of complete pointed metric spaces $\left(\mathcal{X}_{\infty}(t), d_{\infty}(t), x_{\infty}(t)\right)$ such that for each $t \in[0, T]$ the subsequence converges to $\left(\mathcal{X}_{\infty}(t), d_{\infty}(t), x_{\infty}(t)\right)$ in the pointed Gromov-Hausdorff topology.

Proof For simplicity, let's define the notation $\overrightarrow{\mathcal{M}}_{i}(t) \doteqdot\left(\mathcal{M}_{i}, g_{i}(t), p_{i}\right)$ and $\overrightarrow{\mathcal{X}}_{\infty}(t) \doteqdot\left(\mathcal{X}_{\infty}(t), d_{\infty}(t), x_{\infty}(t)\right)$. We shall take a number of subsequences, each of which we shall continue to index by $i$.

Since the Ricci curvatures are bounded below for each time $t \in[0, T]$, at each time we can find a subsequence which converges to a metric space. Instead, let $\mathcal{D}$ be a countable dense subset of $[0, T]$. For each $t_{0} \in \mathcal{D}$ we can find a subsequence of $\overrightarrow{\mathcal{M}}_{i}\left(t_{0}\right)$ converging to a metric space $\overrightarrow{\mathcal{X}}_{\infty}\left(t_{0}\right)$. We can diagonalize to find a subsequence which converges for all $t_{0} \in \mathcal{D}$ since $\mathcal{D}$ is countable. We now show that the subsequence (which we index also by $i$ ) is convergent for all $t \in[0, T]$. 
For each $t \in[0, T]$ there is a subsequence which converges to $\overrightarrow{\mathcal{X}}_{\infty}(t)$. We claim that $\overrightarrow{\mathcal{M}}_{i}(t)$ converges to $\overrightarrow{\mathcal{X}}_{\infty}(t)$ not just for the subsequence, but for all $i \rightarrow \infty$.

Fix $\varepsilon>0$ and $t \in[0, T]$. By Proposition 12 there is a $\delta>0$ such that if $(1+\delta)^{-1} g_{i}\left(t_{0}\right) \leq g_{i}(t) \leq(1+\delta) g_{i}\left(t_{0}\right)$ then $d_{G H}\left(\overrightarrow{\mathcal{M}}_{i}\left(t_{0}\right), \overrightarrow{\mathcal{M}}_{i}(t)\right)<\varepsilon$. This implies by Proposition 8 that

$$
d_{G H}\left(\overrightarrow{\mathcal{X}}_{\infty}\left(t_{0}\right), \overrightarrow{\mathcal{X}}_{\infty}(t)\right) \leq 4 \varepsilon
$$

by taking the limit of the subsequence which converges for $t$. By our assumption, there exists $\eta>0$ such that if $\left|t-t_{0}\right|<\eta$ then

$$
(1+\delta)^{-1} g_{i}\left(t_{0}\right) \leq g_{i}(t) \leq(1+\delta) g_{i}\left(t_{0}\right) .
$$

Since $\mathcal{D}$ is a dense set, we can choose $t_{0} \in \mathcal{D}$ such that $\left|t-t_{0}\right| \leq \eta$. Thus there exists $t_{0} \in \mathcal{D}$ such that

$$
d_{G H}\left(\overrightarrow{\mathcal{M}}_{i}\left(t_{0}\right), \overrightarrow{\mathcal{M}}_{i}(t)\right)<\varepsilon
$$

Finally, since $\overrightarrow{\mathcal{M}}_{i}\left(t_{0}\right)$ converges to $\overrightarrow{\mathcal{X}}_{\infty}\left(t_{0}\right)$ we can find $I$ large enough so that

$$
d_{G H}\left(\overrightarrow{\mathcal{M}}_{i}\left(t_{0}\right), \overrightarrow{\mathcal{X}}_{\infty}\left(t_{0}\right)\right)<\varepsilon
$$

for $i>I$. Hence, using our triangle inequality (Proposition 7) for GromovHausdorff distance, we have for any $\varepsilon>0$ there is an $I>0$ such that if $i>I$ then

$$
d_{G H}\left(\overrightarrow{\mathcal{M}}_{i}(t), \overrightarrow{\mathcal{X}}_{\infty}(t)\right) \leq 2(2(\varepsilon+\varepsilon)+4 \varepsilon)=16 \varepsilon .
$$

Hence $\overrightarrow{\mathcal{M}}_{i}(t) \rightarrow \overrightarrow{\mathcal{X}}_{\infty}(t)$ as $i \rightarrow \infty$.

Note that we haven't explicitly used solutions of the Ricci flow, although solutions of the Ricci flow with bounded curvature would satisfy the premises of the theorem, as shown below. Also note that we haven't really used the upper curvature bound yet. That will be done in the next section when we look at local properties of the limit.

We can now strengthen this result. Since $\left(\mathcal{M}_{i}, g_{i}(t)\right)$ and $\left(\mathcal{M}_{i}, g_{i}(0)\right)$ are homeomorphic by Lipschitz homeomorphisms, we can show that $\mathcal{X}_{\infty}(t)$ is homeomorphic to $\mathcal{X}_{\infty}(0)$.

Proposition 21 If $\left|\frac{\partial}{\partial t} g_{i}(t)[V, V]\right| \leq C g_{i}(t)[V, V]$ for each vector $V$ and for all $t \in[0, T]$ then $\left(\mathcal{X}_{\infty}(t), x_{\infty}(t)\right)$ is homeomorphic to $\left(\mathcal{X}_{\infty}(0), x_{\infty}(0)\right)$ for all $t \in[0, T]$. 
Proof An easy argument shows that the condition on the derivative of the metric implies that

$$
e^{-C^{\prime} t} g_{i}(0) \leq g_{i}(t) \leq e^{C^{\prime} t} g_{i}(0)
$$

where $C^{\prime}$ is a constant depending only on $C$ and dimension (see [22, Section 8] for details). Hence for all $i \in \mathbb{N}$ and $t \in[0, T]$, we must have

$$
e^{-C^{\prime} T} g_{i}(0) \leq g_{i}(t) \leq e^{C^{\prime} T} g_{i}(0) .
$$

This implies that $\left(\mathcal{M}_{i}, g_{i}(t)\right)$ and $\left(\mathcal{M}_{i}, g_{i}(0)\right)$ are uniformly Lipschitz homeomorphic via the identity map, where for each $i$ the identity map is a Lipschitz homeomorphism. By Proposition 9 we must have that $\left(\mathcal{X}_{\infty}(t), d_{\infty}(t), x_{\infty}(t)\right)$ is Lipschitz homeomorphic to $\left(\mathcal{X}_{\infty}(0), d_{\infty}(0), x_{\infty}(0)\right)$, and the homeomorphism takes $x_{\infty}(t)$ to $x_{\infty}(0)$.

Corollary 22 If $\left\{\left(\mathcal{M}_{i}, g_{i}(t), O_{i}\right)\right\}_{i \in \mathbb{N}}$ is a sequence of complete solutions of the Ricci flow for $t \in[0, T]$ such that the curvatures are uniformly bounded then there is a subsequence which converges to a family of complete metric spaces $\left(\mathcal{X}_{\infty}, d_{\infty}(t), x_{\infty}\right)$ in the pointed Gromov-Hausdorff topology.

Proof The Ricci flow with uniformly bounded curvature satisfies the assumption of Theorem 20 and Proposition 21.

\section{Local theory}

In this section we complete the proof of the main theorem. We need to understand the local theory of the limit metric spaces. We proceed according to Fukaya in [14].

Note that we need only look at a neighborhood of the basepoint because of Proposition 13. Fix frames $\mathcal{F}_{i}$ for $T_{p_{i}} \mathcal{M}_{i}$. Take $\phi_{i}=\exp _{p_{i}} \circ \mathcal{F}_{i}$, where the exponential map is the exponential map for the metric $g_{i}(0)$, independent of $t$. Then $\phi_{i}: B(0,1) \rightarrow \mathcal{M}_{i}$, where the ball is taken with respect to the Euclidean distance. Since the $\phi_{i}$ are constant with respect to time and the metrics $g_{i}$ satisfy the Ricci flow equation, it follows that the metrics $\tilde{g}_{i}(t) \doteqdot \phi_{i}^{*} g_{i}(t)$ satisfy the Ricci flow equation. We use Proposition 19. Since $r(t)$ is a decreasing function, $r(T) \leq r(t)$ and hence $B\left(0, r(T)^{2} / 4\right) \subset B_{\tilde{g}_{i}(t)}(0, r(T) / 4) \subset B(0,1 / 4)$.

By Hamilton's lemma (our Lemma 17) all space derivatives of curvature are bounded, and hence all space and time derivatives of the metric are bounded by a fixed metric (say the Euclidean metric), so there is a subsequence which 
converges in $C^{\infty}(B(0,1) \times[0, T])$ to a family of Riemannian metrics $\tilde{g}_{\infty}(t)$. The time derivatives follow from the Ricci flow equation and bounds on the curvature and its derivatives. Because the convergence is $C^{\infty}, \tilde{g}_{\infty}(t)$ satisfies the Ricci flow equation.

We define the pseudogroups $\Gamma_{i}$ in the same way that Fukaya does, as continuous maps that preserve $\phi_{i}$, i.e.

$$
\Gamma_{i}=\left\{\gamma \in C\left(B\left(0, \frac{1}{2}\right), B(0,1)\right): \phi_{i} \circ \gamma=\phi_{i}\right\} .
$$

It is easy to check that $\Gamma_{i}$ are pseudogroups which act as equivalence relations (so the quotient is well-defined) on $B(0,1 / 4)$. Also $\Gamma_{i}$ are independent of $t$ and are isometries of $\left(B\left(0, r(T)^{2}\right), \tilde{g}_{i}(t)\right)$ for each $t \in[0, T]$ (simply because the metrics $\tilde{g}_{i}(t)$ are defined as pullbacks by $\phi_{i}$, which does not depend on $t$ ). This may appear very restrictive and possibly surprising, but it is because the metrics are pulled back by a common local covering map, so $\tilde{g}_{i}$ are constructed to have these symmetries. Furthermore, they converge to a limit pseudogroup $\Gamma_{\infty}$ (see Fukaya [14, p. 9]).

At this point Fukaya shows that $\left(B(0,1 / 2), \Gamma_{i}\right)$ converges to $\left(B(0,1 / 2), \Gamma_{\infty}\right)$ in the equivariant Gromov-Hausdorff topology, and hence $B(0,1 / 2) / \Gamma_{i}$ converges $B(0,1 / 2) / \Gamma_{\infty}$ in the Gromov-Hausdorff topology by [12].

Since $\left(B\left(O_{i}, 1 / 2\right)\right)$ converge in the Gromov Hausdorff topology to $B\left(O_{\infty}, 1 / 2\right)$ and also $B(0,1 / 2) / \Gamma_{i}$ are isometric to $B\left(O_{i}, 1 / 2\right)$, by Proposition 9 $B(0,1 / 2) / \Gamma_{\infty}$ is isometric to $B\left(O_{\infty}, 1 / 2\right)$. This is precisely what happens when $t=0$, however we need to work a little harder when $t \neq 0$. The reason is twofold. Firstly the Euclidean ball $B(0, r)$ is not a metric ball for $\tilde{g}_{i}(t)$ unless $t=0$ since the $\phi_{i}$ is the exponential map for $g_{i}(t)$. Secondly we must be careful, because $\left(B(0,1 / 2), \tilde{g}_{i}(t)\right) / \Gamma_{i}$ may not be isometric to $\left(B_{g_{i}(t)}\left(O_{i}, 1 / 2\right), g_{i}(t)\right)$ but to some other set. However, the Gromov-Hausdorff convergence only really makes good sense on metric balls. Thus we need to be very careful with which sets we use.

Now consider

$$
\mathcal{F}_{i}(t) \doteqdot\left(\phi_{i}\right)^{-1}\left[\overline{B_{g_{i}(t)}\left(p_{i}, \frac{r(T)}{4}\right)}\right] \subset \overline{B(0,1 / 4)}
$$

The $\mathcal{F}_{i}(t)$ form a set of closed subsets of the compact set $\overline{B(0,1 / 4)}$, and so for each $t$ there is a subsequence which converges to $\mathcal{F}_{\infty}(t)$, a closed subset of $\overline{B(0,1 / 2)}$. This is because the closed subsets of a compact set are compact in the Hausdorff topology. We claim that we can find a subsequence such that 
$\mathcal{F}_{i}(t) \rightarrow \mathcal{F}_{\infty}(t)$ for all $t \in[0, T]$ where $\mathcal{F}_{\infty}(t)$ are some closed sets. We can find a subsequence which converges for all time by the following proposition.

Proposition 23 There exist closed sets $\mathcal{F}_{\infty}(t)$ and a subsequence such that for each $t \in[0, T], \mathcal{F}_{i}(t)$ converges to $\mathcal{F}_{\infty}(t)$ in the Hausdorff distance.

Proof Take a countable dense subset $\mathcal{D} \subset[0, T]$. Since for each $t$ there is a subsequence which converges, we can diagonalize so that there is a subsequence which converges for all $t \in \mathcal{D}$. We then show that this sequence converges for all $t \in[0, T]$. Clearly we can define $\mathcal{F}_{\infty}(t)$ from the subsequence which converges. Now since $B_{g_{i}(t)}\left(p_{i}, \frac{r(T)}{4}\right)$ is arbitrarily close to $B_{g_{i}(s)}\left(p_{i}, \frac{r(T)}{4}\right)$ for some $s \in \mathcal{D}$ (due to the facts that $\mathcal{D}$ is dense and we have estimates from Proposition 18) we get convergence for all $t$.

An easy argument (see [16] for details) shows that $\left(\mathcal{F}_{i}(t), \Gamma_{i}\right)$ converges to $\left(\mathcal{F}_{\infty}(t), \Gamma_{\infty}\right)$ in the equivariant Gromov-Hausdorff distance since both converge separately in the Hausdorff distance. Thus $\mathcal{F}_{i}(t) / \Gamma_{i}$ converges to $\mathcal{F}_{\infty}(t) / \Gamma_{\infty}$ in the Gromov-Hausdorff distance by [13, Theorem 2.1]. Since $\overline{B_{g_{i}(t)}\left(p_{i}, \frac{r(T)}{4}\right)}$ must converge to $\overline{B_{d_{\infty}(t)}\left(x_{\infty}, \frac{r(T)}{4}\right)}$ by Proposition 11 we can use Proposition 9 to show that $\mathcal{F}_{\infty}(t) / \Gamma_{\infty}$ is isometric to $\overline{B_{d_{\infty}(t)}\left(x_{\infty}, \frac{r(T)}{4}\right)}$. Let's call that isometry

$$
\xi_{t}: \mathcal{F}_{\infty}(t) / \Gamma_{\infty} \rightarrow \overline{B_{d_{\infty}(t)}\left(x_{\infty}, \frac{r(T)}{4}\right)} .
$$

We need only find the appropriate open set in $\mathcal{X}_{\infty}$ to complete the proof of the main theorem.

Notice that $B\left(0, \frac{r(T)^{2}}{4}\right) \subset \mathcal{F}_{i}(t)$ for all $i$ because

$$
\phi_{i}\left(B\left(0, \frac{r(T)^{2}}{4}\right)\right)=B_{g_{i}(0)}\left(p_{i}, \frac{r(T)^{2}}{4}\right) \subset B_{g_{i}(t)}\left(p_{i}, \frac{r(T)}{4}\right)
$$

for all $i$, so it follows that $B\left(0, \frac{r(T)^{2}}{4}\right) \subset \mathcal{F}_{\infty}(t)$. We claim that

$$
\xi_{t}\left[\pi\left(B\left(0, \frac{r(T)^{2}}{4}\right)\right)\right] \subset B_{d_{\infty}(t)}\left(x_{\infty}, \frac{r(T)}{4}\right)
$$

is open and hence is open in $\mathcal{X}_{\infty}$ where

$$
\pi: B(0,1 / 4) \rightarrow B(0,1 / 4) / \Gamma_{\infty}
$$


is the projection map. Let $y=\xi_{t}[\pi(\tilde{y})]$, where $\tilde{y} \in B\left(0, \frac{r(T)^{2}}{4}\right)$. Since $\xi_{t}$ is an isometry and $\pi$ is a quotient by isometries, we have that

$$
\begin{aligned}
d_{\infty}(t)\left[x_{\infty}, y\right] & =d_{\infty}(t)\left[\xi_{t}[\pi(0)], \xi_{t}[\pi(\tilde{y})]\right] \\
& =\bar{d}_{\tilde{g}_{\infty}(t)}(\pi(0), \pi(\tilde{y})) \\
& \leq d_{g_{\infty}(t)}(0, \tilde{y}) \\
& <\frac{r(T)^{2}}{4} \\
& <\frac{r(T)}{4}
\end{aligned}
$$

and hence $y \in B_{d(t)}\left(x_{\infty}, \frac{r(T)}{4}\right)$. Note, the distance on the quotient is defined as

$$
\bar{d}(\pi(x), \pi(y)) \doteqdot \inf _{\gamma, \gamma^{\prime} \in \Gamma_{\infty}}\left\{d\left(\gamma \cdot x, \gamma^{\prime} \cdot y\right)\right\}
$$

which justifies the third step. Now, $\pi\left(B\left(0, \frac{r(T)^{2}}{4}\right)\right)=B\left(0, \frac{r(T)^{2}}{4}\right) / \Gamma_{\infty}$ is open in $\mathcal{F}_{\infty}(t) / \Gamma_{\infty}$ because

$$
\pi^{-1}\left(B\left(0, \frac{r(T)^{2}}{4}\right) / \Gamma_{\infty}\right)=\bigcup_{\gamma \in \Gamma_{\infty}} \gamma\left[B\left(0, \frac{r(T)^{3}}{4}\right)\right]
$$

which, since all $\gamma \in \Gamma_{\infty}$ are isometries, is the union of open sets and hence open. Thus

$$
\mathcal{U}(t) \doteqdot \xi_{t}\left[\pi\left(B\left(0, \frac{r(T)^{2}}{4}\right)\right)\right] \subset B_{d_{\infty}(t)}\left(x_{\infty}, \frac{r(T)}{4}\right)
$$

is open in $B_{d_{\infty}(t)}\left(x_{\infty}, \frac{r(T)}{4}\right)$, and hence open in $\mathcal{X}_{\infty}$. Thus there is an open set $\mathcal{U}(t) \subset \mathcal{X}_{\infty}$ which is isometric to $B\left(0, \frac{r(T)^{2}}{4}\right) / \Gamma_{\infty}$ with the induced quotient metric of $\tilde{g}_{\infty}(t)$. This completes the proof of the main theorem.

As a corollary, we get Hamilton's compactness theorem. For simplicity, we state it with curvature bound 1 and injectivity radius bound 1 at every point. In fact, we could get Hamilton's full result using the Cheeger-Gromov-Taylor [7] or Cheng-Li-Yau [9] result which bounds how fast the injectivity radius can fall off with distance.

Theorem 24 Let $\left\{\left(\mathcal{M}_{i}, g_{i}(t), p_{i}, \mathcal{F}_{i}\right)\right\}_{i=1}^{\infty}$ be a sequence of complete marked Riemannian manifolds of dimension $n$ evolving by the Ricci flow such that the curvatures are uniformly bounded by 1 and the covariant derivatives of 
the curvature tensor are uniformly bounded, and, in addition, the injectivity radii are uniformly bounded below by 1 . Then there exists a subsequence which converges to a marked $C^{\infty}$ Riemannian manifold $\left(\mathcal{M}_{\infty}, g_{\infty}(t), p_{\infty}, \mathcal{F}_{\infty}\right)$ in $C^{\infty}$ on compact subsets.

Proof (sketch) We use the main theorem (Theorem 3), where the pseudogroups are trivial because the injectivity radii at every point is bounded below by 1 . We now have embeddings $B(0, r(T)) \rightarrow \mathcal{X}_{\infty}$ and we claim that these form a $C^{\infty}$ structure such that the local Riemannian metrics fit together to form a global metric.

For $y \in \mathcal{M}_{i}$ look at the map $\phi_{i, y}: B(0, r(T)) \rightarrow B_{g_{i}}(y, r(T)) \subset \mathcal{M}_{i}$, induced by a frame and the exponential map; now consider the overlaps maps $\phi_{i, y}^{-1} \circ \phi_{i, y^{\prime}}$. These are geodesic coordinates in a fixed metric, so by the curvature bounds we can bound all derivatives of the maps uniformly in $i$. Thus we can take a subsequence so that these maps converge in $C^{\infty}$, where we take countably many points $\left\{y_{i, j}\right\} \subset \mathcal{M}_{i}$ so that

$$
\mathcal{M}_{i}=\bigcup_{j=1}^{\infty} \phi_{i, y_{i, j}}(B(0, r(T))) .
$$

(We used Arzela-Ascoli to see that there exists a convergent subsequence.) The limit maps are smooth transition functions, and thus give $\mathcal{X}$ a smooth structure. Hence $\mathcal{X}$ is a manifold and we can call it $\mathcal{M}_{\infty}$, and define $p_{\infty} \doteqdot x_{\infty}$. By continuity we see that the limit metrics form a tensor on all of $\mathcal{M}_{\infty}$, and hence we have a global limit Riemannian metric $g_{\infty}(t)$ for all $t$ and since it evolves by the Ricci flow in every coordinate patch, $g_{\infty}(t)$ is a solution to the Ricci flow.

\section{$6 \quad$ Examples}

In this section we give two examples of collapsing sequences of Ricci flow. The first gives an example where we do not converge to the Ricci flow on the collapsed manifold. The second is a homogeneous example of what happens to a particular nonsingular solution.

Example 25 $\mathcal{M}_{i}=\left(S^{1} \times S^{1}, d r^{2}+\frac{1}{i} f(r)^{2} d s^{2}\right)$ 
The Gauss curvature of $\mathcal{M}_{i}$ is

$$
K=-\frac{f^{\prime \prime}}{f}
$$

and so the Ricci flow on $\mathcal{M}_{i}$ is

$$
\frac{\partial g_{i}}{\partial t}=2 \frac{f^{\prime \prime}}{f} g_{i} .
$$

Notice that if we project to the first component, this is not stationary, but clearly the initial metrics converge to the standard metric on $S^{1}$. Were this the Ricci flow, $S^{1}$ would be stationary, but the image of the Ricci flow is changing.

In this case we can produce a global covering structure since the exponential map is a covering map, and we see that the structure in the limit is simply the group $\mathbb{R}$ acting on the second component of the universal cover $\mathbb{R} \times \mathbb{R}$ with metric $d \tilde{r}^{2}+f(\tilde{r} \bmod 2 \pi)^{2} d \tilde{s}^{2}$. Note that the two-dimensional Ricci flow converges to a constant curvature metric by [20], and hence the collapsed metric converges to a fixed size metric, the image of $S^{1} \times S^{1}$ under the quotient map.

Example 26 Nonsingular convergence of Nil.

Consider the Ricci flow solution starting with a Nil metric on $\mathbb{R}^{3}$, say start with

$$
g_{0}=A(d z-x d y)^{2}+B d y^{2}+C d x^{2}
$$

and then evolve by the Ricci flow, and call this metric $g(t)$ at time $t$. Let this live on the torus, i.e. we quotient by the isometry group generated by the isometries

$$
\begin{aligned}
(x, y, z) & \rightarrow(x+1, y, z) \\
(x, y, z) & \rightarrow(x, y+1, z) \\
(x, y, z) & \rightarrow(x, y, z+1) .
\end{aligned}
$$

An easy calculation along the lines of [24] gives the Ricci flow solution for the associated ODE to be

$$
\begin{aligned}
& A(t)=\left(2 t+C_{1}\right)^{-1 / 2} \\
& B(t)=C_{2}\left(2 t+C_{1}\right)^{1 / 2} \\
& C(t)=C_{3}\left(2 t+C_{1}\right)^{1 / 2}
\end{aligned}
$$

for some constants $C_{k}$. Note that the solution is nonsingular. Now we may take the following sequence of solutions:

$$
g_{i}(t)=g(i+t)
$$


and consider $\mathcal{M}_{i}=\left(S^{1} \times S^{1} \times S^{1}, g_{i}(t)\right)$. It is easy to see that the sequence converges to two dimensional Euclidean space $\mathbb{E}^{2}$, (noncompact) and the limit of the covers have the metrics $d z^{2}+C_{2} d y^{2}+C_{3} d x^{2}$. We show this by rescaling. Consider the map

$$
\phi_{i}(x, y, z)=\left(\frac{x}{\left(2 i+C_{1}\right)^{1 / 4}}, \frac{y}{\left(2 i+C_{1}\right)^{1 / 4}},\left(2 i+C_{1}\right)^{1 / 4} z\right)
$$

which is a map

$$
\mathbb{R}^{3} / \sim_{2} \rightarrow \mathbb{R}^{3} / \sim_{1}=S^{1} \times S^{1} \times S^{1}
$$

where equivalence $\sim_{1}$ comes from the isometries above and the equivalence $\sim_{2}$ comes from the induced maps

$$
\begin{aligned}
& (x, y, z) \rightarrow\left(x+\left(2 i+C_{1}\right)^{1 / 2}, y, z\right) \\
& (x, y, z) \rightarrow\left(x, y+\left(2 i+C_{1}\right)^{1 / 2}, z\right) \\
& (x, y, z) \rightarrow\left(x, y, z+\left(2 i+C_{1}\right)^{-1 / 2}\right) .
\end{aligned}
$$

These are the groups $\Gamma_{i}$. It is clear that these group actions become translation in the $z$-direction, which is the group $\Gamma_{\infty}$ in the limit as $i \rightarrow \infty$. Now consider

$$
\begin{aligned}
4 \phi_{i}^{*} g_{i}(t)= & \left(2 t+2 i+C_{1}\right)^{-1 / 2}\left[\left(2 i+C_{1}\right)^{1 / 2} d z^{2}-2 x d z d y+\left(2 i+C_{1}\right)^{-1} x^{2} d y^{2}\right] \\
& +C_{2} \frac{\left(2 t+2 i+C_{1}\right)^{1 / 2}}{\left(2 i+C_{1}\right)^{1 / 2}} d y^{2}+C_{3} \frac{\left(2 t+2 i+C_{1}\right)^{1 / 2}}{\left(2 i+C_{1}\right)^{1 / 2}} d x^{2}
\end{aligned}
$$

so for $t=0$, as $i \rightarrow \infty$ we get convergence to a Euclidean space with metric $d z^{2}+C_{2} d y^{2}+C_{3} d x^{2}$ on the universal cover. Actually, since

$$
\lim _{i \rightarrow \infty} \frac{\left(2 t+2 i+C_{1}\right)^{1 / 2}}{\left(2 i+C_{1}\right)^{1 / 2}}=1
$$

we get that it converges to this for each time $t$., The manifolds converge in Gromov-Hausdorff to the quotient, which is a steady state Euclidean plane $\mathbb{E}^{2}$.

\section{References}

[1] D Burago, Y Burago, S Ivanov, A course in metric geometry, volume 33 of Graduate Studies in Mathematics, American Mathematical Society, Providence, RI (2001)

[2] M Carfora, J Isenberg, M Jackson, Convergence of the Ricci flow for metrics with indefinite Ricci curvature, J. Differential Geom. 31 (1990) 249-263 
[3] M Carfora, A Marzuoli, Model geometries in the space of Riemannian structures and Hamilton's flow, Classical Quantum Gravity 5 (1988) 659-693

[4] J Cheeger, K Fukaya, M Gromov, Nilpotent structures and invariant metrics on collapsed manifolds, J. Amer. Math. Soc. 5 (1992) 327-372

[5] J Cheeger, M Gromov, Collapsing Riemannian manifolds while keeping their curvature bounded. I, J. Differential Geom. 23 (1986) 309-346

[6] J Cheeger, M Gromov, Collapsing Riemannian manifolds while keeping their curvature bounded. II, J. Differential Geom. 32 (1990) 269-298

[7] J Cheeger, M Gromov, M Taylor, Finite propagation speed, kernel estimates for functions of the Laplace operator, and the geometry of complete Riemannian manifolds, J. Differential Geom. 17 (1982) 15-53

[8] J Cheeger, X Rong, Collapsed Riemannian manifolds with bounded diameter and bounded covering geometry, Geom. Funct. Anal. 5 (1995) 141-163

[9] S Y Cheng, P Li, S-T Yau, On the upper estimate of the heat kernel of a complete Riemannian manifold, Amer. J. Math. 103 (1981) 1021-1063

[10] B Chow, D Glickenstein, $\mathbf{P}$ Lu, Collapsing sequences of solutions to the Ricci flow on 3-manifolds with almost nonnegative curvature, arXiv:math.DG/0305228

[11] B Chow, D Knopf, P Lu, Hamilton's injectivity radius estimate for sequences with almost nonnegative curvature operators, Comm. Anal. Geom. 10 (2002) 1116-1140

[12] K Fukaya, Theory of convergence for Riemannian orbifolds, Japan. J. Math. (N.S.) 12 (1986) 121-160

[13] K Fukaya, Collapsing Riemannian manifolds to ones of lower dimensions, J. Differential Geom. 25 (1987) 139-156

[14] K Fukaya, A boundary of the set of the Riemannian manifolds with bounded curvatures and diameters, J. Differential Geom. 28 (1988) 1-21

[15] K Fukaya, Collapsing Riemannian manifolds to ones with lower dimension. II, J. Math. Soc. Japan 41 (1989) 333-356

[16] D Glickenstein, Precompactness of solutions to the Ricci flow and a maximum principle for combinatorial Yamabe flow, Ph.D. thesis, University of California at San Diego (2003)

[17] R E Greene, $\mathbf{H} \mathbf{W u}$, Lipschitz convergence of Riemannian manifolds, Pacific J. Math. 131 (1988) 119-141

[18] M Gromov, Metric structures for Riemannian and non-Riemannian spaces, Birkhäuser Boston Inc., Boston, MA (1999), based on the 1981 French original [MR 85e:53051], With appendices by M. Katz, P. Pansu and S. Semmes, Translated from the French by S. M. Bates

[19] R S Hamilton, Three-manifolds with positive Ricci curvature, J. Differential Geom. 17 (1982) 255-306 
[20] R S Hamilton, The Ricci flow on surfaces, from: "Mathematics and general relativity (Santa Cruz, CA, 1986)", Contemp. Math. 71, Amer. Math. Soc., Providence, RI (1988) 237-262

[21] R S Hamilton, A compactness property for solutions of the Ricci flow, Amer. J. Math. 117 (1995) 545-572

[22] R S Hamilton, The formation of singularities in the Ricci flow, from: "Surveys in differential geometry, Vol. II (Cambridge, MA, 1993)", Internat. Press, Cambridge, MA (1995) 7-136

[23] R S Hamilton, J Isenberg, Quasi-convergence of Ricci flow for a class of metrics, Comm. Anal. Geom. 1 (1993) 543-559

[24] J Isenberg, M Jackson, Ricci flow of locally homogeneous geometries on closed manifolds, J. Differential Geom. 35 (1992) 723-741

[25] D Knopf, Quasi-convergence of the Ricci flow, Comm. Anal. Geom. 8 (2000) $375-391$

[26] D Knopf, K McLeod, Quasi-convergence of model geometries under the Ricci flow, Comm. Anal. Geom. 9 (2001) 879-919

[27] $\mathbf{P} \mathbf{L u}$, A compactness property for solutions of the Ricci flow on orbifolds, Amer. J. Math. 123 (2001) 1103-1134

[28] G Perelman, The entropy formula for the Ricci flow and its geometric applications, arXiv:math.DG/0211159

[29] G Perelman, The Ricci flow with surgery on three-manifolds, arXiv:math.DG/0303109

[30] S Peters, Convergence of Riemannian manifolds, Compositio Math. 62 (1987) 3-16

[31] P Petersen, Convergence theorems in Riemannian geometry, from: "Comparison geometry (Berkeley, CA, 1993-94)", Cambridge Univ. Press, Cambridge (1997) 167-202

[32] A Petrunin, X Rong, W Tuschmann, Collapsing vs. positive pinching, Geom. Funct. Anal. 9 (1999) 699-735

[33] L S Pontryagin, Topological groups, Translated from the second Russian edition by Arlen Brown, Gordon and Breach Science Publishers, Inc., New York (1966)

[34] X Rong, Collapsed manifolds with pinched positive sectional curvature, J. Differential Geom. 51 (1999) 335-358

[35] T Shioya, T Yamaguchi, Collapsing three-manifolds under a lower curvature bound, J. Differential Geom. 56 (2000) 1-66

[36] T Yamaguchi, Collapsing and pinching under a lower curvature bound, Ann. of Math. (2) 133 (1991) 317-357 